\title{
Reversible Data Hiding Based on Adaptive Block Selection Strategy
}

\author{
Dan Huang, Guangdong Provincial Key Laboratory of Information, Security Technology, Sun Yat-sen University, \\ Guangzhou, China \\ Fangjun Huang, School of Data and Computer Science, Sun Yat-sen University, Guangzhou, China
}

\begin{abstract}
Recently, a reversible data hiding (RDH) method was proposed based on local histogram shifting. This method selects the peak bin of the local histogram as a reference and expands the two neighboring bins of the peak bin to carry the message bits. Since the peak bin keeps unchanged during the embedding process, the neighboring bins can be easily identified at the receiver end, and the original image can be restored completely while extracting the embedded data. In this article, as an extension of the algorithm, the authors propose an RDH scheme based on adaptive block selection strategy. Via a new block selection strategy, those blocks of the carrier image may carry more message bits whereas introducing less distortion will take precedence over data hiding. Experimental results demonstrate that higher visual quality can be obtained compared with the original method, especially when the embedding rate is low.
\end{abstract}

\section{KEYWORDS}

Block Selection, Histogram Shifting, Localization, Reversible Data Hiding, Visual Quality

\section{INTRODUCTION}

Reversible data hiding $(\mathrm{RDH})$ technique aims to embed message bits into a carrier image by slightly modifying its pixels, and more importantly, it can completely restore the original carrier image while extracting the embedded data from the marked image. As a special case of information hiding, RDH can be applied to some areas when the reversibility is desirable, such as medical and military image processing.

Many RDH algorithms have been proposed so far. Basically, there are three fundamental strategies: lossless compression (Celik, Sharma, Tekalp, \& Saber, 2005; Fridrich, Goljan, \& Du, 2002), difference expansion (Hu, Lee, \& Li, 2009; Sachnev, Kim, Nam, Suresh, \& Shi, 2009; Tai, Yeh, \& Chang, 2009; Tian, 2003), and histogram shifting (Huang, Qu, Kim, \& Huang, 2016; Ni, Shi, Ansari, \& Su, 2006; Xuan, Shi, Ni, Chai, Cui, \& Tong, 2007). The lossless compression-based methods apply lossless compression to the carrier image, and utilize the statistical redundancy to create a free space for data hiding. This strategy has received less attention recently, since it cannot provide large embedding capacity and may lead to severe degradation in image visual quality. The difference expansion (DE) strategy was firstly proposed by Tian (Tian, 2003), where the carrier image is divided into pixel pairs and the difference value of two pixels in a pair is expanded to carry one message bit. The histogram shifting (HS) strategy was proposed by Ni et al. (Ni, Shi, Ansari, \& Su, 2006). The method utilizes the peak bin of the original histogram of the carrier image for data hiding. After that, many RDH algorithms based on HS strategy have been proposed. Recent research has demonstrated that the HS

This article, originally published under IGI Global's copyright on January 1, 2020 will proceed with publication as an Open Access article starting on January 27, 2021 in the gold Open Access journal, International Journal of Digital Crime and Forensics (converted to gold Open Access January 1, 2021), and will be distributed under the terms of the Creative Commons Attribution License (http://creativecommons.org/ licenses/by/4.0/) which permits unrestricted use, distribution, and production in any medium, provided the author of the original work and original publication source are properly credited. 
strategy can also be applied to the difference histogram and the prediction-error histogram $(\mathrm{Li}, \mathrm{Li}$, \& Yang, 2013; Li, Yang, \& Zeng, 2011; Li, Zhang, Gui, \& Yang, 2013; Li, Li, Li, \& Yang, 2013; Li, Zhang, Gui, \& Yang, 2017; Ou, Li, Zhao, Ni, \& Shi, 2013; Peng, Li, \& Yang, 2014; Wu, \& Sun, 2014; Huang, Huang, \& Shi, 2016), which can achieve larger embedding capacity and better image visual quality.

In 2015, Pan et al. (Pan, Hu, Ma, \& Wang, 2015) proposed a novel RDH algorithm based on local histogram shifting. In this method, the peak bin of the local histogram of the carrier image is selected as the reference bin, and the two neighboring bins of the peak bin are expanded to carry message bits. Although Pan et al.'s method achieves large embedding capacity, it may result in unsatisfactory image visual quality, because some of the blocks that will introduce much distortion may be selected for data hiding, even if the embedding rate is low.

In this paper, we propose a new adaptive RDH scheme based on Pan et al.'s method. The main idea of the proposed method is to design an adaptive block selection strategy, and thus the blocks with higher embedding capacities and less distortion will take precedence for data hiding. Compared with Pan's method, the proposed method keeps the same maximum embedding capacity, and achieves better image visual quality at the same payload. Extensive experimental results demonstrate the effectiveness of the proposed method.

The rest of this paper is organized as follows. In Section 2, Pan et al.'s scheme is introduced. Section 3 presents the proposed method in detail. The experimental results are given in Section 4, and the conclusion is drawn in Section 5.

\section{PAN ET AL.'S METHOD}

In 2015, Pan et al. proposed a RDH method based on local histogram shifting. The embedding procedure is as below. First, divide the pre-processed (the pre-processing process will be introduced at the end of this section) carrier image $I$ into non-overlapping blocks with the size of $s \times s$. Then, for each block $B$, its local histogram is obtained. Suppose that $B_{p}$ is the grayscale value of the pixels associated with the peak bin of the local histogram. The embedding algorithm of Pan et al.'s RDH scheme can be described as Equation (1),

$$
B_{i, j}^{\prime}= \begin{cases}B_{i, j}-1 & \text { if } 1 \leq B_{i, j} \leq B_{p}-2 \\ B_{i, j}-b & \text { if } B_{i, j}=B_{p}-1 \\ B_{i, j} & \text { if } B_{i, j}=B_{p} \\ B_{i, j}+b & \text { if } B_{i, j}=B_{p}+1 \\ B_{i, j}+1 & \text { if } B_{p}+2 \leq B_{i, j} \leq 254\end{cases}
$$

where $b=\{0,1\}$ denotes the message bit to be embedded, $B_{i, j}$ represents the pixel value at the position $(i, j)$ in the block $B$, and $B_{i, j}^{\prime}$ represents the pixel value at the position $(i, j)$ in the embedded block $B^{\prime}$.

The message extraction and image restoration can be described as Equation (2) and Equation (3),

$$
b^{\prime}=\left\{\begin{array}{l}
0 \quad \text { if } B_{i, j}^{\prime}=B_{p}+1 \text { or } B_{p}-1 \\
1 \quad \text { if } B_{i, j}^{\prime}=B_{p}+2 \text { or } B_{p}-2
\end{array}\right.
$$




$$
B_{i, j}^{*}= \begin{cases}B_{i, j}^{\prime}+1 & \text { if } 0 \leq B_{i, j}^{\prime} \leq B_{p}-2 \\ B_{i, j}^{\prime} & \text { if } B_{i, j}^{\prime}=B_{p} \text { or } B_{p} \pm 1 \\ B_{i, j}^{\prime}-1 & \text { if } B_{p}+2 \leq B_{i, j}^{\prime} \leq 255\end{cases}
$$

where $b^{\prime}$ and $B_{i, j}^{*}$ represent the extracted message bit and the restored pixel value, respectively. Since the peak bin of each block remains unchanged after data embedding, the neighboring bins can be easily identified at the receiver end, and thus the original image can be restored completely while extracting the embedded data.

Note that in Pan et al.'s method, for those pixels whose values are equal to 0 or 255 , the overflow or underflow will be introduced while embedding the message bits. To avoid it, the carrier image needs to be pre-processed prior to the data embedding. The pixels valued 0 will be changed to 1 , and the pixels valued 255 will be changed to 254 . To record the pre-processed pixels, a location map with the same size as the carrier image is generated by assigning 1 to the location of a pre-processed pixel, and 0 to that of an unchanged one. The location map is then compressed as side information and embedded into the carrier image along with the message bits.

\section{PROPOSED METHOD}

\subsection{Motivation of the Proposed Method}

Pan et al.'s method has some advantages, for example, high embedding capacity can be easily obtained, and no extra information (the peak point and zero point) needs to be transmitted to the receiver end for message extraction and image restoration. Moreover, in other RDH algorithms, such as Ni et al.'s method (Ni, Shi, Ansari, \& Su, 2006), the global histogram of the marked image is not continuous anymore because of the great changes caused by the histogram shifting during data embedding. While in Pan et al.'s method, the localization can equally redistribute those great changes into the local histograms, which can help the global histogram of the marked image keep being continuous as that of the original carrier image. However, Pan et al.'s method still has some limitations. For example, it may greatly influence the visual quality of the carrier image even if the embedding rate is low.

One reason for the unsatisfactory visual quality of Pan et al.'s method is that it randomly selects the image blocks for data hiding. However, we find that selecting the blocks randomly is not a reasonable strategy, since embedding message bits into different blocks may result in different distortion. We explain this issue with examples as follows. In Figure 1, we illustrate the local histograms of two different blocks in "Lena" image with the size of $8 \times 8$. For ease of explanation, the occurrence of pixels with the value $B_{p}$ in the block $B$ is represented by $N_{B p}$, and the occurrence of pixels with the value $B_{p} \pm 1$ is represented by $N_{B p \pm 1}$. In Figure 1(a), the peak point $B_{p}$ is located at 135 . As seen, in this block, there are 4 pixels with value $B_{p}, 1$ pixel with value $B_{p}-1$, and no pixel with value $B_{p}+1$. According to Pan et al.'s embedding algorithm, when this block is selected for data hiding, only one message bit can be embedded, and there are 59 invalid shifting. However, for the image block corresponding to Figure 1(b), $B_{p}$ is located at 207, and the value of $N_{B p}$ is 20, the value of $N_{B p \pm 1}$ is 31 . If this block is selected for data hiding, 31 message bits can be embedded, and there are only 13 invalid shifting. Base on the above analysis, if the blocks selected for data hiding are similar to the first block, much more distortion will be introduced, and thus the visual quality of the marked image may be greatly degraded even if the embedding rate is low. Thus, in this paper, we try to design a new block selection strategy that can adaptively select the image blocks for data hiding. 
Figure 1. Histograms of two different blocks in "Lena" image with size of $8 \times 8$. (a) One block with low embedding capacity and many invalid shifting. (b) One block with high embedding capacity and few invalid shifting.

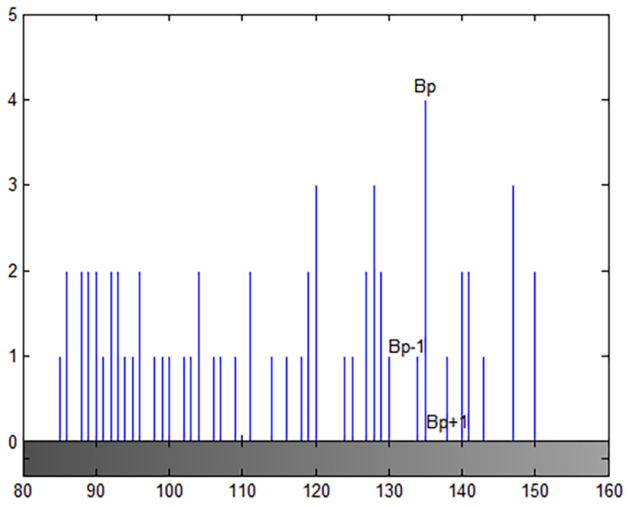

(a)

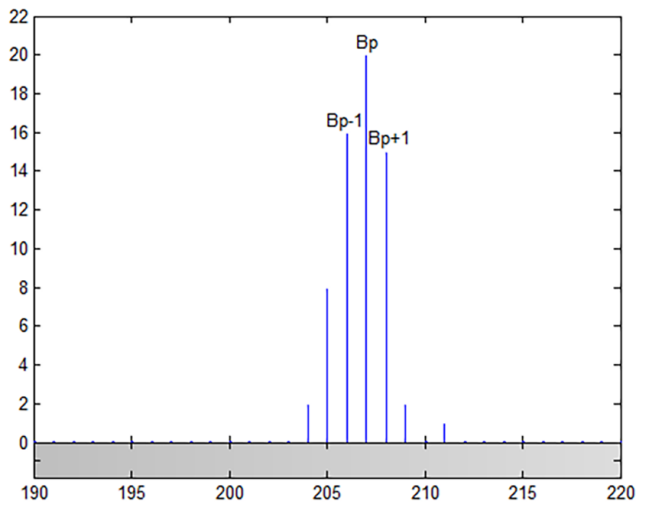

(b)

In our block selection strategy, the main idea is to find image blocks that can carry more message bits with less distortion. Based on this idea, one consideration is to optimize the embedding performance by adaptively selecting embedding blocks according to the embedding capacity of each block. Namely, those blocks with more pixels valued $B_{p} \pm 1$ will take precedence for data hiding, meaning that the selected blocks can embed more message bits. However, if this block selection strategy is adopted, the receiver will not be able to differentiate the marked block and the original block since the value of $N_{B p \pm 1}$ is not preserved in the marked image. Thus, a location map is needed to record the selected embedding blocks, which will introduce more invalid shifting, decrease the embedding capacity, and degrade the image visual quality. Especially when the block size is small, the location map is large.

As we know, the pixel values of the natural image are continuously changed in the spatial domain. That is, most of the pixel values in the divided block may be similar or even the same, especially when the block is located in the flat area. Thus in the local histogram of an image block, the occurrence of pixels with the value $B_{p}$ (i.e., $N_{B p}$ ) may have a close relationship with the occurrence of pixels with the values $B_{p} \pm 1$ (i.e., $N_{B p \pm 1}$ ). To validate this assumption, we conduct the following test. We divide the original "Lena" image into non-overlapping blocks with the sizes of $8 \times 8,16 \times 16,32 \times 32$ and $64 \times 64$, respectively. The testing results corresponding to different block sizes are shown in Figure 2. In Figure 2(a)-(d), the horizontal axes represent the values of $N_{B p}$, and the vertical axes represent the values of $N_{B p \pm 1}$, i.e., the embedding capacity of each block. It is observed from Figure 2 that the embedding capacity has an approximate linear relationship with $N_{B p}$. Since the value of $N_{B p}$ in each block keeps unchanged after data hiding, it can be utilized to adaptively select the blocks for data hiding. In our algorithm, the blocks with more pixels valued $B_{p}$ will take precedence for data hiding.

\subsection{The Proposed Adaptive Block Selection Method}

As introduced in Section 1, the original carrier image is pre-processed, a location map $L M$ is generated to record the locations of those pre-processed pixels and compressed to reduce its length. After pre-processing, we divide the pre-processed image $I$ into non-overlapping $s \times s$ blocks, and try to embed data into those blocks with larger value of $N_{B p}$. Note that in each block, the occurrence 
Figure 2. The approximate linear relationship between the peak occurrence and embedding capacity of each block with different block sizes. (a) Lena, $8 \times 8$. (b) Lena, $16 \times 16$. (c) Lena, $32 \times 32$. (d) Lena, $64 \times 64$.

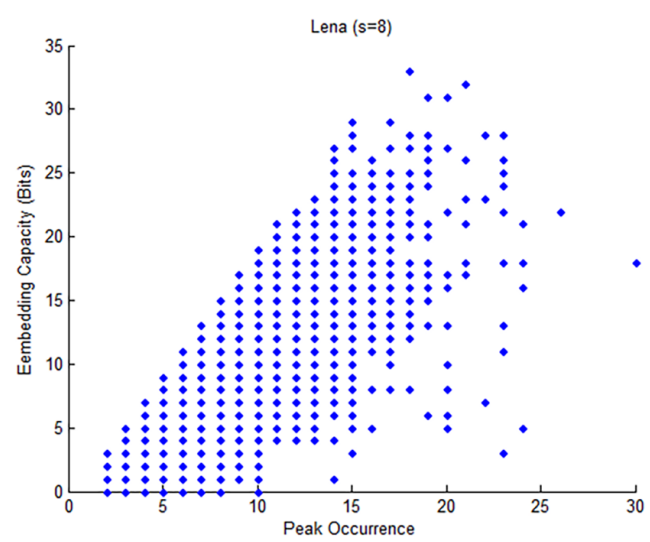

(a)

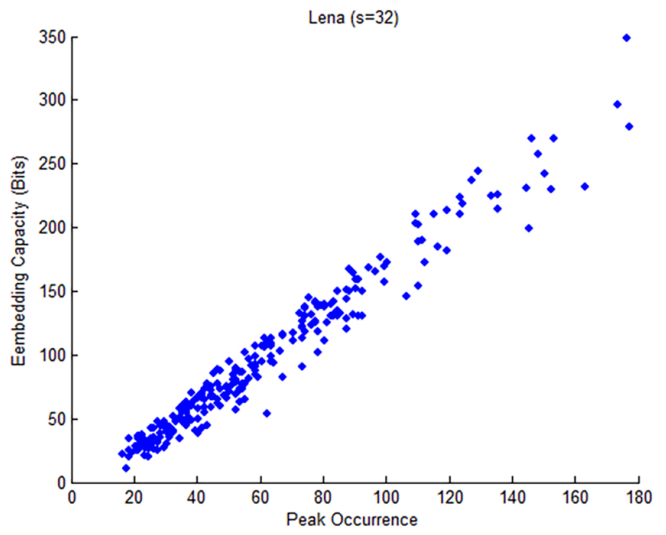

(c)

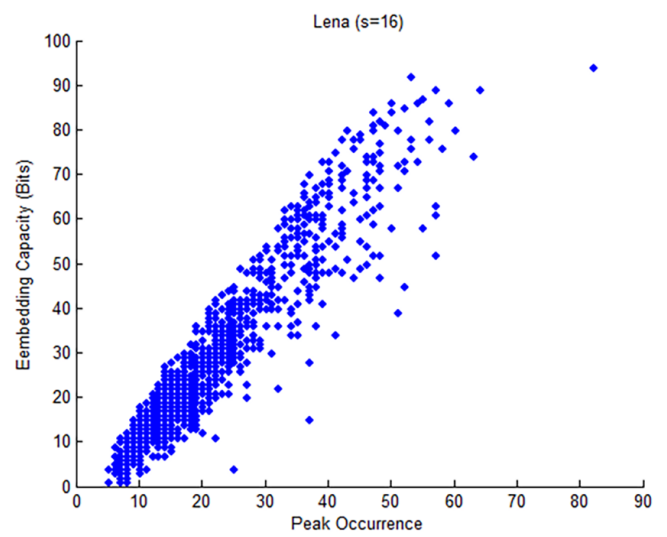

(b)

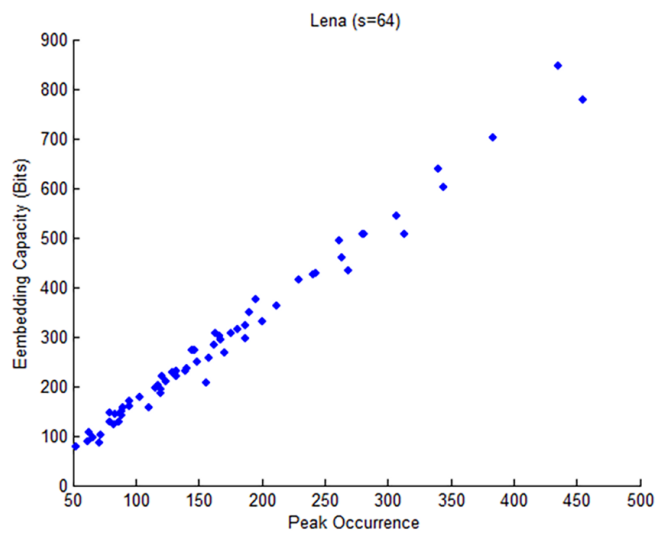

(d)

of pixels with the value $B_{p}$ is represented by $N_{B p}$. The blocks are divided into two groups. The first group includes all the blocks whose $N_{B p}$ values are not less than a threshold value $T_{Z}$, and the second group includes the rest of the blocks. The sum of all $N_{B p \pm 1} \mathrm{~s}$ in the first group (note that each block has one $N_{B p \pm 1}$ ) is represented by $S$, and $S$ is the total embedding capacity of the first group. The threshold value $T_{Z}$, which is used to select the embedding blocks in our algorithm, is determined by

$T_{Z}=\underset{T}{\operatorname{argmax}}\left\{S \geq\left(L+M+l_{1}+l_{2}+l_{3}\right)\right\}$

where $1 \leq T \leq s^{2}, L$ is the number of message bits to be embedded, $M$ is the number of compressed $L M$ bits, $l_{1}$ is the number of bits needed to represent the threshold value $T_{Z}, l_{2}$ is the number of bits needed to represent the value of $L$, and $l_{3}$ is the number of bits needed to represent the value of $M$. In Equation (4), $T_{Z}$ is taken as the largest positive integer in the range $\left[1, s^{2}\right]$ such 
that the payload can be successfully embedded. We can select $l_{1}=12, l_{2}=24$ and $l_{3}=14$, which are acceptable for most applications.

Note that in our method, only the blocks in the first group are utilized for data hiding, and those blocks in the second group are kept untouched in the embedding process.

\subsection{The Efficiency of Our New Block Selection Strategy}

In Pan et al.'s RDH scheme, the inner pixels with values $B_{p} \pm 1$ are expanded to carry message bits, whereas the outer pixels with values $B_{p} \pm 2, B_{p} \pm 3, \ldots$, and so on, are shifted so that the inner and outer regions of the pixel histogram are still kept separate from each other after data hiding. Obviously, the outer pixels do not carry any information, but they need to be shifted; this invalid shifting will degrade the visual quality of the marked image.

In order to demonstrate the efficiency of our new block selection strategy, some statistical results corresponding to eight standard grayscale images with the size of $512 \times 512$ are shown in Table 1 . The images are illustrated in Figure 3. Each image is divided into $N$ blocks, and the blocks are sorted according to the value of $N_{B p}$. The first part includes $N / 2$ blocks with larger values of $N_{B p}$, and the second part includes the rest of the blocks. For all of the 8 images, the average number of inner pixels $N_{\text {in }}$, the average number of outer pixels $N_{\text {out }}$, and the value of $N_{\text {in }} / N_{\text {out }}$ in each part are shown in Table 1. It is observed that in the first part, the ratio of $N_{\text {in }}$ to $N_{\text {out }}$ ranges from $12 \%$ to $25 \%$. However, in the second part, the ratio of $N_{\text {in }}$ to $N_{\text {out }}$ ranges from $4 \%$ to $6 \%$. It indicates that embedding the message bits into blocks in the first part will lead to less invalid shifting. Thus, our proposed adaptive block selection strategy is efficient in preserving the visual quality of the marked image.

Figure 3. The test images. (a) Lena (b) Baboon (c) Lake (d) Boat (e) Goldhill (f) Airplane (g) Tiffany (h) Barbara.

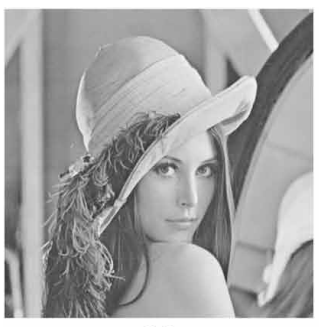

(a)

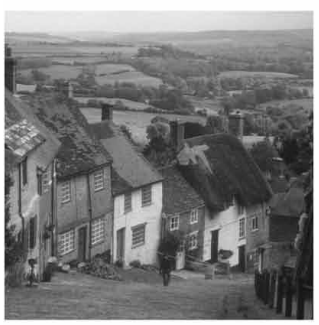

(e)

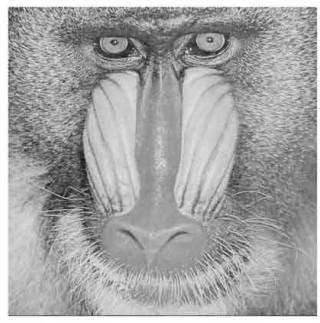

(b)

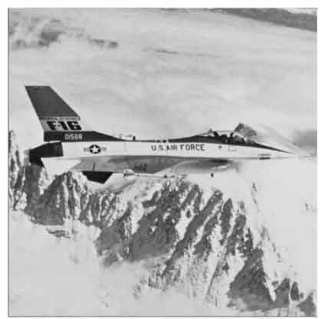

(f)

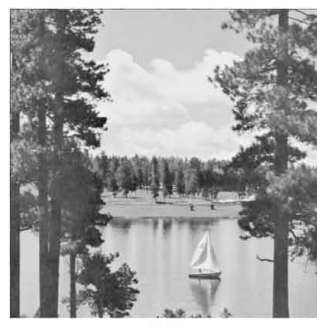

(c)

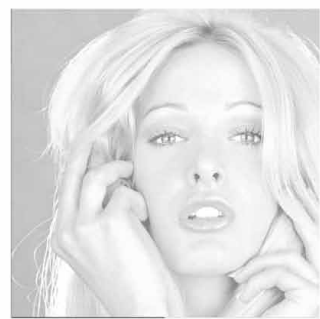

(g)

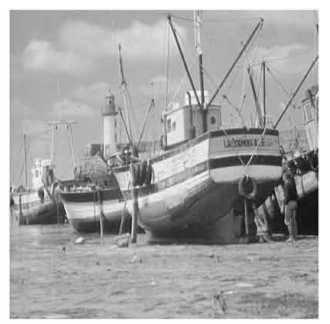

(d)

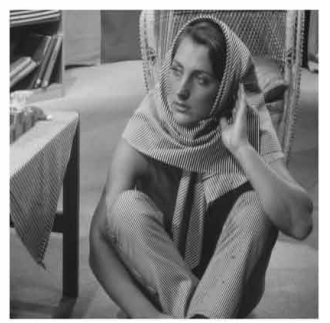

(h) 
Table 1. The statistics corresponding to inner and outer pixels

\begin{tabular}{|c|c|c|c|c|c|c|}
\hline \multirow{2}{*}{ Block size } & \multicolumn{3}{|c|}{ The first part } & \multicolumn{3}{c|}{ The second part } \\
\cline { 2 - 7 } & $N_{\text {in }}$ & $N_{\text {out }}$ & $N_{\text {in }} / N_{\text {out }}$ & $N_{\text {in }}$ & $N_{\text {out }}$ & $N_{\text {in }} / N_{\text {out }}$ \\
\hline $8 \times 8$ & 22049 & 87481 & 0.2520 & 7164 & 114344 & 0.0626 \\
\hline $16 \times 16$ & 20044 & 95796 & 0.2092 & 6533 & 118600 & 0.0551 \\
\hline $32 \times 32$ & 16773 & 103165 & 0.1626 & 5835 & 121115 & 0.0482 \\
\hline $64 \times 64$ & 13207 & 109871 & 0.1202 & 5158 & 122713 & 0.0420 \\
\hline
\end{tabular}

\subsection{Embedding, Extraction and Restoration Steps}

1. Embedding steps

Step 1. Pre-process the carrier image, construct the location map $L M$, and attach the compressed $L M$ to the message bits.

Step 2. Divide the pre-processed carrier image $I$ into non-overlapping blocks with the size of $s \times s$. As described in Section 3.2, obtain the threshold value $T_{Z}$ according to Equation (4).

Step 3. Shuffle all the blocks with a secret key, and scan the resulting blocks sequentially. Embed the threshold value $T_{Z}$ (represented with $l_{1}$ bits) into the first visited block according to Equation (1). Note that in this step, if the first visited block has value $N_{B p}$ which is not less than $T_{Z}$, it will continue to embed some other information bits according to Step 4. Otherwise, only the threshold value $T_{Z}$ is embedded into this block.

Step 4. According to the sequential order, successively embed the message length $L$ (represented with $l_{2}$ bits), the compressed $L M$ length $M$ (represented with $l_{3}$ bits), the message bits and the compressed $L M$ into the pre-processed carrier image. Note that in this step, if the value $N_{B p}$ of the current block is not less than $T_{Z}$, the information bits are embedded according to Equation (1). Otherwise, do nothing and continue to visit the next block according to the scanning order. Here the current block is refer to the block which is being visited.

2. Extraction and restoration steps

Step 1. Divide the marked image $I^{\prime}$ into non-overlapping blocks with the size of $s \times s$.

Step 2. Shuffle the blocks with the same secret key as that used in the embedding process and scan the resulting blocks sequentially. Extract the threshold value $T_{Z}$ (i.e., the first $l_{1}$ bits) from the first visited block according to Equation (2) and restore the pre-processed image according to Equation (3). Note that if the first visited block has value $N_{B p}$ which is not less than $T_{Z}$, continuously extract the other information bits according to Step 3. Otherwise, only the threshold value $T_{Z}$ is extracted from the block.

Step 3. According to the sequential order, successively extract the message length $L$ (i.e., the first $l_{2}$ bits), the compressed $L M$ length $M$ (i.e., the following $l_{3}$ bits), $L$ message bits and the compressed $L M$ from the embedded blocks according to Equation (2) and restore the pre-processed image according to Equation (3). Note that in this step, if the current block has value $N_{B p}$ which is not less than $T_{Z}$, the information bits are extracted according to Equation (3) and the pre-processed image are restored according to Equation (4). Otherwise, do nothing and continue to visit the next block according to the scanning order.

Step 4. According to $L M$, recover the original pixels modified in the pre-processing procedure. Consequently, the original carrier image can be recovered completely. 
Note that in the proposed algorithm, all the pixels with value $B_{p}$ remain unchanged in the embedding process. The receiver can easily locate the blocks utilized for data hiding according to $T_{Z}$, and then extract the embedded data and restore the carrier image. Moreover, no extra location map is needed to record the blocks selected for data hiding.

\section{EXPERIMENTAL RESULTS}

In all our experiments, the secret message bits are randomly generated, and aforementioned eight images are used in our testing. To evaluate the performance of the proposed new block selection strategy, Pan et al.'s method is included for comparison. The peak signal to noise ratio (PSNR) is used as a measurement to evaluate the visual quality of the marked image, which is calculated between the original image and the marked one.

The test images are divided into non-overlapping blocks with the size of $s \times s \quad(\mathrm{~s}=8,16,32$ and 64). The experimental results are shown in Figure 4-7 (we independently repeat the experiments 10 times with different secret keys and show the average results in this figure), where the horizontal axes represent the pure embedding payloads and the vertical axes represent the average PSNR values. The name of the test image and the block size are specified in the title of each sub-figure. It is observed from Figure 4-7 that the average PSNR values obtained by the proposed method are generally larger than those obtained by Pan et al.'s method. For example, for the "Airplane" image, if the block size is $8 \times 8$ and payload is 10000 bits, the PSNR value that obtained by Pan et al.'s method is about 56.12 $\mathrm{dB}$, while the PSNR value can be increased to $62.15 \mathrm{~dB}$ if our new block selection strategy is adopted. Obviously, the proposed adaptive RDH method has better performance in image visual quality compared with Pan et al.'s method; and the high embedding capacity still remains. Besides, we make a comparison with Li et al.'s method (Li, Yang, \& Zeng, 2011) in Figure4. We can see that the proposed method can achieve better performance than Li et al.'s in some cases. Moreover, the proposed method can also keep the global histogram of the marked image being continuous as that of the original carrier image. As seen, our new block selection strategy retains the main advantages of Pan et al.'s method (e.g., the high embedding capacity, the continuous histogram of the embedded image) and meanwhile the visual quality of the marked image can be greatly improved.

\section{CONCLUSION}

In this paper, we proposed a RDH scheme based on adaptive block selection strategy. Based on our analyses and experiments, we find that the occurrence of pixels with value $B_{p}$ (i.e., $N_{B p}$ ) has an approximate linear relationship with the embedding capacity of the block, and thus propose a new block selection strategy based on the value of $N_{B p}$. Via using our new block selection strategy, those blocks that can carry more message bits whereas introduce less distortion will take precedence for data hiding. Moreover, no extra location map of the selected embedding blocks needs to be transmitted to the receiver end for data extraction and image restoration. The proposed block selection strategy can retain the main advantages of Pan et al.'s method; meanwhile, the visual quality of the marked image can be significantly improved. In the future, we will try to adapt the proposed method for RDH in color images and JPEG images.

\section{ACKNOWLEDGMENT}

This work is supported in part by the National Natural Science Foundation of China (61772572), in part by the NSFC-NRF Scientific Cooperation Program (61811540409), and in part by the Natural Science Foundation of Guangdong Province of China (2017A030313366). Fangjun Huang is the corresponding author for this paper. 
Figure 4. The visual qualities corresponding to different pure embedding payloads for these eight images with different block sizes $(8 \times 8,16 \times 16,32 \times 32,64 \times 64)$. (a) Lena (b) Baboon
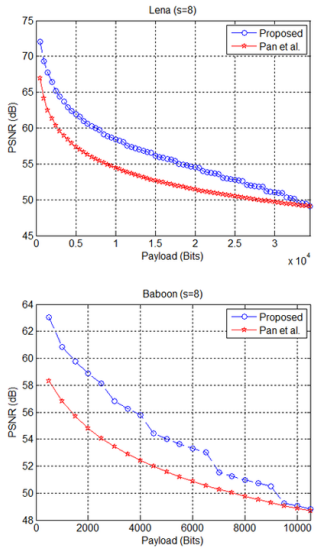
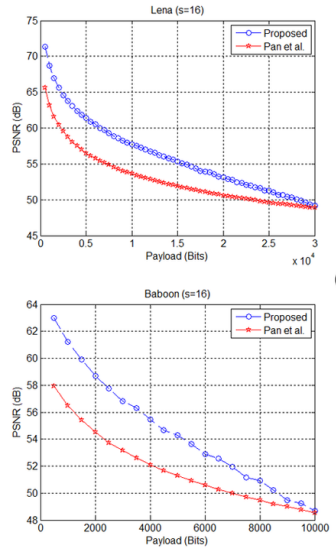

(a)
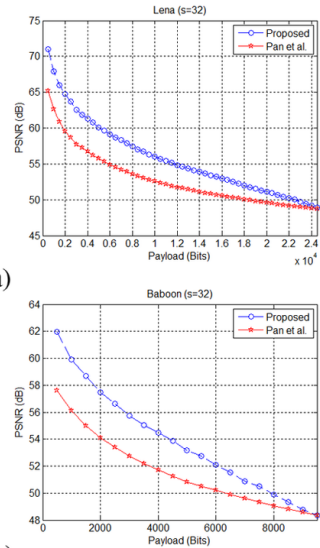
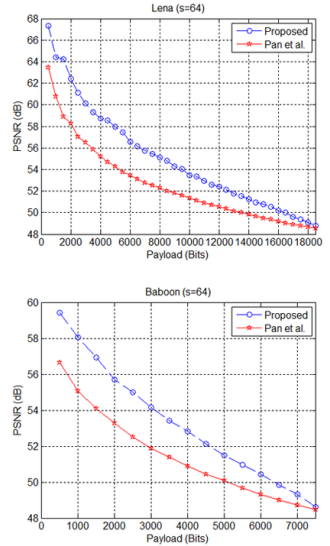

(b)

Figure 5. The visual qualities corresponding to different pure embedding payloads for these eight images with different block sizes $(8 \times 8,16 \times 16,32 \times 32,64 \times 64)$. ((c) Lake (d) Boat
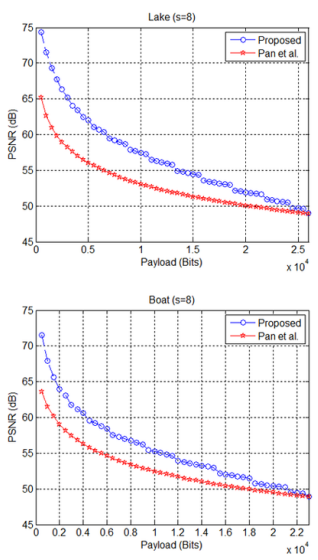
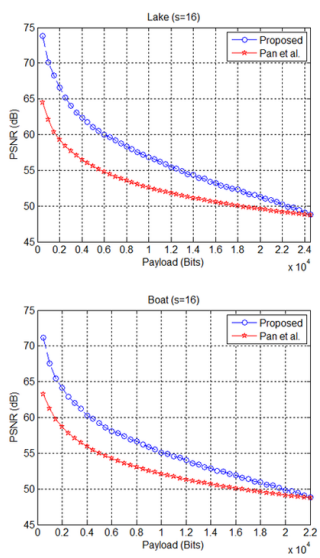

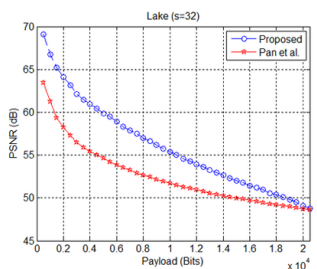

(c)

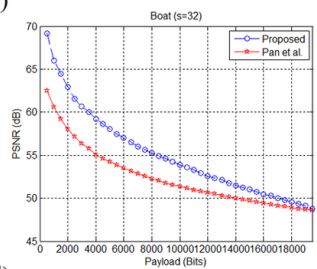

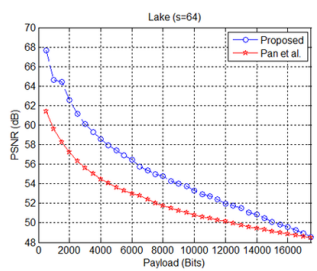

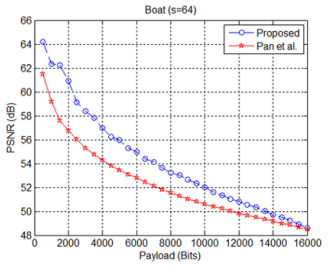

(d)

Figure 6 . The visual qualities corresponding to different pure embedding payloads for these eight images with different block sizes $(8 \times 8,16 \times 16,32 \times 32,64 \times 64)$. (e) Goldhill (f) Airplane
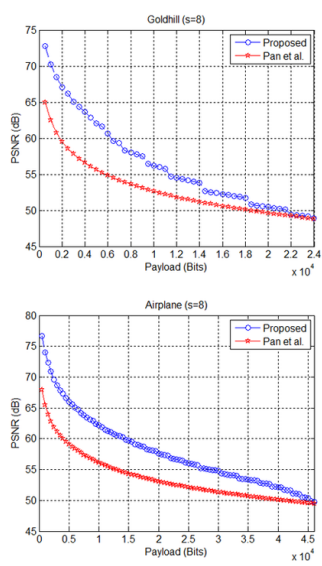
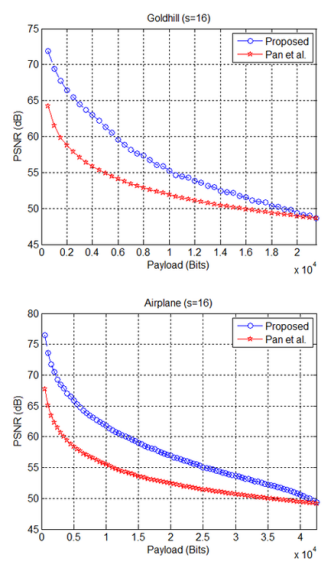

(e)
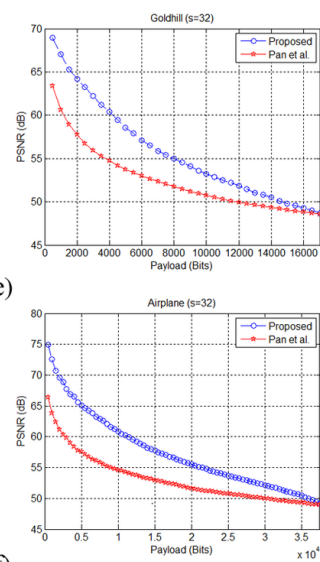
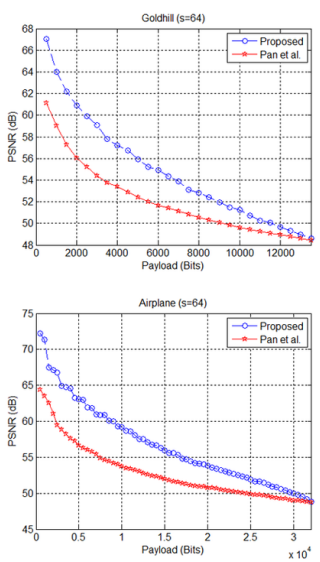
Figure 7. The visual qualities corresponding to different pure embedding payloads for these eight images with different block sizes $(8 \times 8,16 \times 16,32 \times 32,64 \times 64)$. (g) Tiffany (h) Barbara.
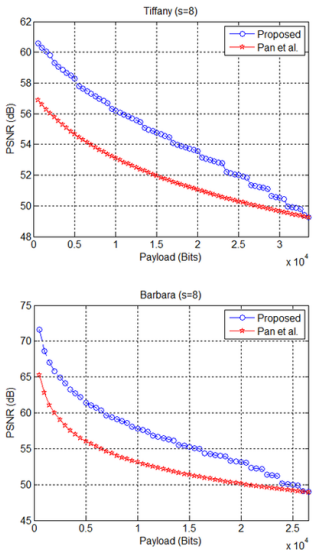
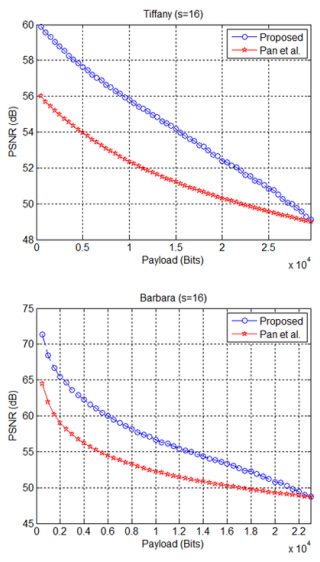

(g)
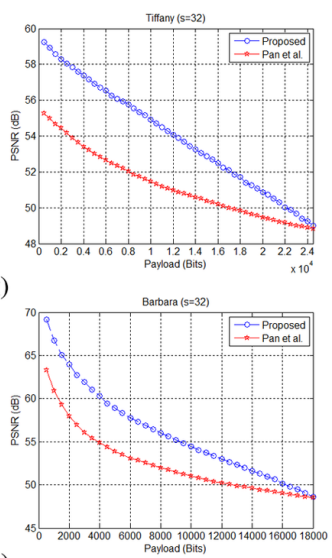
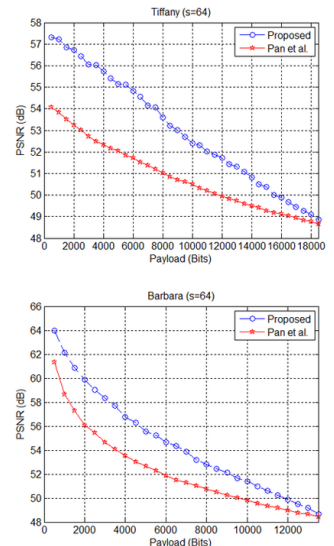

(h) 


\section{REFERENCES}

Celik, M. U., Sharma, G., Tekalp, A. M., \& Saber, E. (2005). Lossless generalized-LSB data embedding. IEEE Transactions on Image Processing, 14(2), 253-266. doi:10.1109/TIP.2004.840686 PMID:15700530

Fridrich, A. J., Goljan, M., \& Du, R. (2002). Lossless data embedding for all image formats. Proceedings of SPIE, 4675, 572-583. doi:10.1117/12.465317

Hu, Y., Lee, H. K., \& Li, J. (2009). DE-Based reversible data hiding with improved overflow location map. IEEE Transactions on Circuits and Systems for Video Technology, 19(2), 250-260. doi:10.1109/TCSVT.2008.2009252

Huang, F., Qu, X., Kim, H. J., \& Huang, J. (2016). Reversible data hiding in JPEG images. IEEE Transactions on Circuits and Systems for Video Technology, 26(9), 1610-1621. doi:10.1109/TCSVT.2015.2473235

Huang, F. H. J., \& Shi, Y. Q. (2016). New Framework for Reversible Data Hiding in Encrypted Domain. IEEE Transactions on Information Forensics and Security, 11(12), 2777-2789. doi:10.1109/TIFS.2016.2598528

Li, J., Li, X., \& Yang, B. (2013). Reversible data hiding scheme for color image based on prediction-error expansion and cross-channel correlation. Signal Processing, 93(9), 2748-2758. doi:10.1016/j.sigpro.2013.01.020

Li, X., Li, J., Li, B., \& Yang, B. (2013). High-fidelity reversible data hiding scheme based on pixel-valueordering and prediction-error expansion. Signal Processing, 93(1), 198-205. doi:10.1016/j.sigpro.2012.07.025

Li, X., Yang, B., \& Zeng, T. (2011). Efficient reversible watermarking based on adaptive prediction-error expansion and pixel selection. IEEE Transactions on Image Processing, 20(12), 3524-2533.

Li, X., Zhang, W., Gui, X., \& Yang, B. (2013). A novel reversible data hiding scheme based on two-dimensional difference-histogram modification. IEEE Transactions on Information Forensics and Security, 8(7), 1091-1100. doi:10.1109/TIFS.2013.2261062

Li, X., Zhang, W., Gui, X., \& Yang, B. (2017). Efficient reversible data hiding based on multiple histograms modification. IEEE Transactions on Information Forensics and Security, 10(9), 2016-2027.

Ni, Z., Shi, Y. Q., Ansari, N., \& Su, W. (2006). Reversible data hiding. IEEE Transactions on Circuits and Systems for Video Technology, 16(3), 354-362. doi:10.1109/TCSVT.2006.869964

Ou, B., Li, X., Zhao, Y., Ni, R., \& Shi, Y. Q. (2013). Pairwise prediction-error expansion for efficient reversible data hiding. IEEE Transactions on Image Processing, 22(12), 5010-5021.

Pan, Z., Hu, S., Ma, X., \& Wang, L. (2015). Reversible data hiding based on local histogram shifting with multilayer embedding. Journal of Visual Communication and Image Representation, 31(C), 64-74. doi:10.1016/j. jvcir.2015.05.005

Peng, F., Li, X., \& Yang, B. (2014). Improved PVO-based reversible data hiding. Digital Signal Processing, 25(2), 255-265. doi:10.1016/j.dsp.2013.11.002

Sachnev, V., Kim, H. J., Nam, J., Suresh, S., \& Shi, Y. Q. (2009). Reversible watermarking algorithm using sorting and prediction. IEEE Transactions on Circuits and Systems for Video Technology, 19(7), 989-999. doi:10.1109/TCSVT.2009.2020257

Tai, W. L., Yeh, C. M., \& Chang, C. C. (2009). Reversible data hiding based on histogram modification of pixel differences. IEEE Transactions on Circuits and Systems for Video Technology, 19(6), 906-910. doi:10.1109/ TCSVT.2009.2017409

Tian, J. (2003). Reversible data embedding using a difference expansion. IEEE Transactions on Circuits and Systems for Video Technology, 13(8), 890-896. doi:10.1109/TCSVT.2003.815962

Wu, X., \& Sun, W. (2014). High-capacity reversible data hiding in encrypted images by prediction error. Signal Processing, 104(6), 387-400. doi:10.1016/j.sigpro.2014.04.032

Xuan, G., Shi, Y. Q., Ni, Z., Chai, P., Cui, X., \& Tong, X. (2007). Reversible data hiding for JPEG images based on histogram pairs. In Proceedings of the International Conference on Image Analysis and Recognition (pp.715-727). Springer-Verlag. doi:10.1007/978-3-540-74260-9_64 
Dan Huang is a master student in Guangdong Provincial Key Laboratory of Information Security Technology, Sun Yat-sen University, Guangzhou, China.

Fangjun Huang joined the School of Data and Computer Science at Sun Yat-Sen University since 2005. He received his B.S. degree from Nanjing University of Science and Technology, China, in 1995, and the M.S. and Ph.D. degrees from Huazhong University of Science and Technology, China, in 2002 and 2005, respectively. From June of 2009 to June of 2010, he was a Postdoctoral Researcher at New Jersey Institute of Technology, NJ, USA. From August of 2013 to August of 2014, He was a Korea Foundation for Advanced Studies (KFAS) scholar at Korea University, Seoul, Korea. His research interests include reversible data hiding, steganography, steganalysis, and digital forensics. 